\section{Prevalência e fatores associados a sintomas de ansiedade em uma coorte de gestantes atendidas em um centro de saúde do município do Rio de Janeiro}

\section{Prevalence of anxiety symptoms and associated factors in a cohort of pregnant women attending a health care center in the municipality of Rio de Janeiro}

Daniele Marano Rocha Araújo 1

Alice Helena de Resende Nóra Pacheco 2 Adriano Marçal Pimenta 3 Gilberto Kac 4

\section{Abstract}

Objectives: to determine the prevalence and the association between socio-demographic, obstetric and nutritional variables and anxiety symptoms in pregnant women.

Methods: the study involved cohorts with five follow-up stages. A cross-sectional analysis was carried out of 151 pregnant women. The presence or absence of anxiety was treated as the dependent variable, which was measured between 19 and 21 weeks of gestation. The State-Trait Anxiety Inventory (STAIT) scale was used to evaluate the anxiety trait. The socio-demographic, obstetric and nutritional data obtained using a questionnaire were treated as independent variables. The statistical analysis was performed using a multivariate Poisson regression with robust variance.

Results: the mean age was 25 years and mean level of schooling nine years. The prevalence of anxiety was $64.9 \%$ (95\% CI: 56.7-72.5). The anxiety trait was associated with age between 18 and 24.9 years $(P R=1.35$; 95\%CI: $1.06-1.71)$ and $1-8$ years of schooling ( $P R=1.39$; 95\%CI: 1.11-1.73).

Conclusions: it was observed that the anxiety trait was highly prevalent in this sample of pregnant women whose progress was followed at a health care center. Knowledge of the factors associated with the anxiety is of fundamental importance in allowing the health team to intervene in a timely and appropriate fashion in a pregnant woman's treatment.

Key words Anxiety, Age, Educational status, Pregnancy

\section{Resumo}

Objetivos: determinar a prevalência e a associação entre variáveis sócio-demográficas, obstétricas, nutricionais e sintomas de ansiedade em gestantes.

Métodos: trata-se de estudo prospectivo com cinco ondas de seguimento. A presente análise é transversal e foi realizada com 151 gestantes. A variável dependente foi a presença ou ausência de ansiedade na gestação, medida entre a $19^{a}$ e $21^{a}$ semana de gestação. Utilizou-se o Inventário de Ansiedade Traço-Estado (IDATE) para avaliar a ansiedade traço (a-traço). As variáveis independentes foram extraídas de um questionário com dados sócio-demográficos, obstétricos e nutricionais. A análise estatística foi realizada por meio da técnica de regressão multivariada de Poisson com variância robusta.

Resultados: as gestantes tinham em média 25 anos e nove anos de escolaridade. A prevalência de ansiedade foi de 64,9\% (IC95\%: 56,7-72,5). No modelo multivariado observou-se que mulheres mais jovens (entre 18 e 24,9 anos, $R P=1,35$; IC95\%: 1,06-1,71) e de menor escolaridade (um a oito anos, $R P=1,39$; IC95\%: 1,111,73) apresentaram maior chance de desenvolver ansiedade.

Conclusões: a prevalência de ansiedade foi elevada nesta amostra de gestantes acompanhadas em uma unidade básica de saúde. O conhecimento dos fatores associados à ansiedade é de fundamental importância para que a equipe multidisciplinar possa intervir de forma precoce e adequada no tratamento das gestantes. Palavras-chave Ansiedade, Idade, Escolaridade, Gravidez 


\section{Introdução}

A gestação é vista como um momento de transição, extremamente significativo durante a vida da mulher e que exige inúmeras adaptações. 1-3 Acredita-se que durante a gravidez a mulher está mais vulnerável ao desenvolvimento de transtornos de ansiedade. ${ }^{4}$ Esses transtornos podem acarretar prejuízos para a gestante, o feto e, às vezes, até para o cônjuge. Sendo assim, o entendimento dos fatores associados à ansiedade durante a gestação é de grande importância, já que é uma oportunidade para a equipe multidisciplinar prevenir, detectar e tratar tais transtornos. 4

Estudos recentes revelaram que os transtornos de ansiedade sub-diagnosticados e não tratados em gestantes podem levar a graves conseqüências materno-fetais, 5 tais como depressão pós-parto, 6 baixo peso ao nascer, ${ }^{7}$ prematuridade, ${ }^{8}$ redução do índice apgar, ${ }^{9}$ entre outros. Em recente revisão de literatura, Araújo et al.10 observaram que a ansiedade na gestação foi associada à prematuridade e/ou ao baixo peso ao nascer. Os valores de razão de chance e risco relativo variaram de 1,08 a 2,31.

Os fatores que geralmente influenciam negativamente no binômio materno-fetal têm sua gênese no período pré-concepcional ou pré-natal. No entanto, observa-se que os estudos não enfocam os fatores associados à ansiedade durante a gestação. Segundo Falcone et al.,4 a atuação multiprofissional deve levar em consideração a interação de variáveis como história pessoal, período gestacional, características sociais, culturais e econômicas vigentes e qualidade da assistência pré-natal.

Considerando a magnitude da prevalência de ansiedade durante a gestação e os riscos a ela associados, este estudo teve como objetivos determinar a prevalência de sintomas de ansiedade em gestantes e avaliar a associação entre variáveis sócio-demográficas, obstétricas, nutricionais e a ansiedade, com base em dados provenientes de um estudo prospectivo conduzido em uma unidade básica de saúde.

\section{Métodos}

O presente trabalho consiste em uma análise transversal com dados coletados na segunda onda de seguimento de um estudo prospectivo com cinco pontos de observação, realizado no Posto de Saúde Madre Tereza de Calcutá (PSMTC), localizado na Ilha do Governador, no município do Rio de Janeiro, Brasil. As gestantes foram captadas no serviço de pré-natal do PSMTC, no período de junho de 2005 a fevereiro de 2007. Todas as grávidas contatadas foram esclarecidas sobre o objetivo da pesquisa e sua metodologia.

Os critérios de elegibilidade para a inclusão no estudo foram: ter entre 8 e 13 semanas de gestação, ter entre 18 e 40 anos de idade, não apresentar gestação gemelar, estar livre de doenças crônicas não transmissíveis, estar livre de doenças infecto-parasitárias e residir na área adstrita ao estudo.

Foram incluídas na primeira entrevista do seguimento 247 gestantes entre a $8^{\text {a }}$ e $13^{\text {a }}$ semana de gestação. No entanto, na presente análise apenas 151 mulheres foram incluídas, pelo fato do desfecho (a-traço) ter sido investigado entre a $19^{\mathrm{a}} \mathrm{e}$ $21^{\text {a }}$ semana gestacional (segunda entrevista do seguimento). $\mathrm{O}$ padrão de perdas foi avaliado levando-se em consideração a distribuição da taxa final de seguimento (relação entre número de gestantes que iniciaram e concluíram acompanhamento).

A variável dependente do estudo é a ansiedade na gestação. Essa variável foi investigada por meio do Inventário de Ansiedade Traço-Estado (IDATE) de Spielberguer et al.,11 por entrevistadores treinados e técnicas padronizadas. O IDATE foi traduzido, adaptado e validado para o Brasil por Biaggio et al.,12 e baseia-se na concepção dualística proposta por Cattell e Scheier,13 que distingue a ansiedade em dois conceitos: traço de ansiedade (atraço) e estado de ansiedade (a-estado). A consistência interna da versão brasileira foi avaliada por meio do coeficiente alpha de Cronbach, e foram obtidos os valores de 0,93 para o sexo masculino e 0,87 para o sexo feminino.

O IDATE é composto por duas escalas distintas para medir os dois conceitos de ansiedade. Tal inventário consiste em uma escala com 40 itens, 20 correspondentes à ansiedade traço e 20 à ansiedade estado. ${ }^{14}$ Existem quatro alternativas de resposta com valores que variam de um a quatro pontos. Assim, para cada escala, a pontuação mínima alcançada é de 20 pontos e a máxima é de 80 pontos. Quanto maior a pontuação, maior a chance de o indivíduo apresentar ansiedade.

A escala de ansiedade traço requer que o sujeito descreva como ele geralmente se sente.14,15 Os escores dessa escala são menos sensíveis a mudanças decorrentes de situações ambientais e permanecem relativamente constantes no tempo. ${ }^{11}$ Nessa escala, o indivíduo tem as seguintes opções de resposta: quase nunca, às vezes, freqüentemente e quase sempre.12,16 Há itens nessa escala que indicam uma direção contrária à ansiedade e por isso devem ser invertidos, ou seja, recodificados. 15 
Neste estudo, foram selecionados e analisados apenas os itens referentes à ansiedade traço, por permitirem melhor comparação com resultados de outros trabalhos. Foram caracterizadas como gestantes ansiosas aquelas que obtiveram pontuação acima de 40, como empregado nos estudos de Rondo et al. 17 e Teixeira et al. 18

A partir do elenco de variáveis coletadas pela pesquisa, as seguintes co-variáveis foram incluídas na análise: a) variáveis sócio-demográficas: idade da mãe (18-24,9, 25-40,9 anos), renda familiar total $(<1,1$ a 2,3 a $5, \geq 6$ salários mínimos), escolaridade (1-8 anos, $\geq 9$ anos de estudo), cor de pele, segundo classificação do entrevistador (branca, preta), estado marital (casada, em união, não vive em união), trabalho durante a gravidez (sim, não); b) variáveis de estilo de vida: hábito de fumar (sim, não); c) variáveis obstétricas: aborto prévio (sim, não), baixo peso ao nascer prévio ( $\operatorname{sim}$, não) e prematuridade prévia (sim, não) e d) variáveis relacionadas ao estado nutricional materno pré-gestacional: peso prégestacional $(\mathrm{kg})$, estatura $(\mathrm{m})$ e Índice de Massa Corporal (IMC) pré-gestacional: peso $(\mathrm{kg}) /$ estatura $\left(\mathrm{m}^{2}\right)$.

Inicialmente, investigou-se a distribuição de cada co-variável da amostra com objetivo de definir as melhores categorias. A renda familiar foi estabelecida pelo salário mínimo vigente $(\mathrm{R} \$ 350,00)$; a escolaridade pelo número de anos de educação formal completados, ficando assim categorizada em menor de nove anos (nível básico) e nove ou mais anos (nível médio e superior); a idade abaixo de 25 anos e maior ou igual a 25 anos, e o IMC foi dividido em abaixo de $26,0 \mathrm{~kg} / \mathrm{m}^{2}$ e acima ou igual a $26,0 \mathrm{~kg} / \mathrm{m}^{2}$. A comparação entre os grupos de gestantes ansiosas e não-ansiosas foi realizada por meio da diferença de médias de variáveis contínuas, empregando-se o teste $t$ de Student.

Nessa mesma fase da análise, calculou-se a prevalência de ansiedade segundo cada uma das covariáveis descritas acima. As prevalências foram comparadas por meio do teste do qui-quadrado para proporções. Na análise bivariada, utilizou-se como medida de efeito a Razão de Prevalência (RP) bruta, com intervalo de confiança de 95\% (IC95\%).

Como a variável dependente (ansiedade) apresenta distribuição binária e o desfecho tem ocorrência transversal, o procedimento de análise multivariada escolhido foi o de regressão de Poisson com variância robusta. ${ }^{19}$ Dessa forma, todas as variáveis com valores de $p \leq 0,25$ na análise bivariada foram selecionadas para o modelo multivariado, com o objetivo de evitar a exclusão de variáveis potencialmente importantes e controlar para a presença de confundimento residual. A variável estado marital foi mantida no modelo multivariado independente da significância estatística uma vez que a mesma apresentou perda seletiva entre o primeiro e o segundo seguimento. $\mathrm{Na}$ análise multivariada foram utilizados os testes da razão de máxima verossimilhança e de Wald para verificar associação. Também foram testadas as correlações e as interações entre as variáveis do modelo final, enquanto a qualidade do mesmo foi obtida por meio da aplicação do teste da bondade (goodness-of-fit test), pela análise dos resíduos e pelo cálculo do coeficiente de determinação $\left(\mathrm{R}^{2}\right)$. O nível de significância estatística estabelecido para a análise nesta etapa foi de $5 \%$.

O estudo foi aprovado pelo Comitê de Ética em Pesquisa da Secretaria Municipal de Saúde do Rio de Janeiro.

\section{Resultados}

As perdas de seguimento se devem ao fato de haver somente 151 mulheres que tinham idade gestacional entre 19 e 21 semanas à época da segunda entrevista. A Tabela 1 apresenta resultados entre as mulheres seguidas e as perdidas. Observa-se que elas diferiam apenas em relação ao estado marital, com uma maior freqüência de solteiras entre as seguidas. A magnitude das perdas ao final do seguimento foi de $38,8 \%$.

A Tabela 2 apresenta as características gerais da amostra. Observa-se que houve diferença significativa em relação à idade das gestantes ansiosas (25,2 anos) e não-ansiosas (27,2 anos). Em relação à escolaridade, não houve diferença significativa entre gestantes ansiosas (3,9 anos) e não-ansiosas (8,8 anos). Não foi verificada diferença significativa entre as médias de renda familiar e das variáveis antropométricas estatura, IMC e peso pré-gestacional entre gestantes ansiosas e nãoansiosas.

A Tabela 3 apresenta dados sobre a distribuição de freqüência, de prevalência e da razão de prevalência bruta para ansiedade traço, segundo variáveis selecionadas. A prevalência global de ansiedade foi de 64,9\% (IC95\%: 56,7-72,5) (dados não apresentados em tabela). As maiores prevalências de ansiedade traço foram observadas em mulheres com escolaridade entre um e oito anos $(77,8 \%)$, fumantes $(77,8 \%)$, que vivem em união $(74,4 \%)$, com idades entre $18-24$ anos $(72,3 \%)$ e que não trabalhavam $(71,0 \%)$.

$\mathrm{Na}$ análise bivariada, as seguintes variáveis foram selecionadas para o modelo multivariado: idade (18 a 24,9 anos, RP=1,29; IC95\%: 1,01-1,66), 
Tabela 1

Distribuição de freqüência para variáveis selecionadas entre perdas, seguimento completo e taxa final de seguimento em gestantes usuárias de uma unidade básica de saúde. Município do Rio de Janeiro, 2005 a 2007.

\begin{tabular}{|c|c|c|c|c|c|}
\hline Variáveis & $\begin{array}{l}\text { Número inicial } \\
\text { de observações }\end{array}$ & $\begin{array}{c}\text { Perdas de } \\
\text { seguimento }\end{array}$ & $\begin{array}{l}\text { Seguimento } \\
\text { completo }\end{array}$ & $\begin{array}{c}\text { Taxa final de } \\
\text { seguimento (\%) }\end{array}$ & Valor $p^{*}$ \\
\hline Idade (anos) & & & & & 0,970 \\
\hline $18,0+25$ & 136 & 53 & 83 & 61,0 & \\
\hline $25,0+41$ & 111 & 43 & 68 & 61,3 & \\
\hline Renda (salário mínimo)** & & & & & 0,640 \\
\hline$<1$ & 24 & 11 & 13 & 54,6 & \\
\hline $1-2$ & 141 & 57 & 84 & 59,6 & \\
\hline $3-5$ & 63 & 22 & 41 & 65,1 & \\
\hline$\geq 6$ & 17 & 5 & 12 & 70,6 & \\
\hline Escolaridade (anos) & & & & & 0,106 \\
\hline $1-8$ & 113 & 50 & 63 & 55,8 & \\
\hline$\geq 9$ & 129 & 44 & 85 & 65,9 & \\
\hline Estado marital & & & & & 0,049 \\
\hline Casada & 54 & 24 & 30 & 55,6 & \\
\hline Vive em união & 136 & 58 & 78 & 57,4 & \\
\hline Não vive em união & 56 & 14 & 42 & 75,0 & \\
\hline Cor da pele (observada) & & & & & 0,290 \\
\hline Branca & 63 & 21 & 42 & 66,7 & \\
\hline Preta & 181 & 74 & 107 & 59,1 & \\
\hline Baixo peso ao nascer (prévio & & & & & 0,634 \\
\hline Sim & 17 & 8 & 9 & 52,9 & \\
\hline Não & 122 & 50 & 72 & 59,0 & \\
\hline Prematuridade (prévia) & & & & & 0,311 \\
\hline Sim & 19 & 10 & 9 & 47,4 & \\
\hline Não & 124 & 50 & 74 & 59,7 & \\
\hline Álcool & & & & & 0,230 \\
\hline Sim & 31 & 9 & 22 & 71,0 & \\
\hline Não & 216 & 87 & 129 & 59,7 & \\
\hline Fumo & & & & & 0,912 \\
\hline Sim & 29 & 11 & 18 & 62,1 & \\
\hline Não & 218 & 85 & 133 & 61,0 & \\
\hline IMC pré-gestacional $\left(\mathrm{kg} / \mathrm{m}^{2}\right)$ & & & & & 0,907 \\
\hline$\leq 25,9$ & 176 & 68 & 108 & 61,4 & \\
\hline$\geq 26,0$ & 71 & 28 & 43 & 60,6 & \\
\hline
\end{tabular}

* Valor $p$ refere-se ao teste de qui-quadrado para proporções; ** Salário mínimo da época=R 350,00 ; IMC=Índice de Massa Corporal

escolaridade (um a oito anos de estudo, $\mathrm{RP}=1,35$; IC95\%: 1,08-1,68), estado marital (vive em união, $\mathrm{RP}=1,19$; IC95\%: 1,02-1,40), fumo (sim, RP=1,23; IC95\%: 0,93-1,63) e trabalho durante a gestação (RP=1,17; IC95\%: 0,93-1,47) (Tabela 3).

Após controle para fatores de confusão pela análise multivariada, as seguintes variáveis permaneceram associadas à ansiedade traço: idade (18 a 24,9 anos, RP=1,35; IC95\%: 1,06-1,71) e escolaridade (um a nove anos de estudo, $\mathrm{RP}=1,39$; IC95\%: 1,11 - 1,73) (Tabela 4). 
Características sócio-demográficas e antropométricas de gestantes ansiosas e não-ansiosas. Município do Rio de Janeiro, 2005 a 2007.

\begin{tabular}{|c|c|c|c|c|c|c|c|c|c|c|c|}
\hline \multirow[t]{2}{*}{ Variáveis } & \multicolumn{5}{|c|}{ Ansiosas* } & \multicolumn{5}{|c|}{ Não-ansiosas ** } & \multirow[t]{2}{*}{ Valor $p$} \\
\hline & $\mathrm{n}$ & Média & Mínimo & Máximo & $\mathrm{IC} 95 \%$ & $\mathrm{n}$ & Média & Mínimo & Máximo & IC95\% & \\
\hline Idade (anos) & 98 & 25,20 & 18,12 & 40,30 & $14,65-35,75$ & 53 & 27,20 & 18,26 & 40,70 & $15,33-39,05$ & 0,043 \\
\hline Escolaridade (anos) $* * *$ & 98 & 3,90 & 1,00 & 8,00 & $0,66-8,46$ & 50 & 8,79 & 1,00 & 8,00 & $35,11-52,70$ & 0,120 \\
\hline Renda familiar (R\$) & 97 & $1.051,12$ & 150,005 & $5.000,00$ & $580,30-682,60$ & 53 & $1.002,80$ & 0,00 & $2.700,00$ & $230,00-468,40$ & 0,713 \\
\hline Estatura $(\mathrm{m})$ & 98 & 1,59 & 1,46 & 1,76 & $1,50-1,70$ & 53 & 1,60 & 1,45 & 1,75 & $1,90-1,70$ & 0,474 \\
\hline IMC (kg/m²) & 98 & 23,81 & 17,04 & 35,85 & $15,03-32,60$ & 53 & 23,78 & 18,42 & 37,93 & $15,83-31,73$ & 0,972 \\
\hline Peso pré-gestacional $(\mathrm{kg})$ & 98 & 60,50 & 41,20 & 98,10 & $35,98-85,04$ & 53 & 60,90 & 44,50 & 94,10 & $39,15-82,65$ & 0,848 \\
\hline
\end{tabular}

*Ansiosas - IDATE (Inventário de Ansiedade Traço-Estado)=>40; ** Não-ansiosas - IDATE $\leq 40 ;$ *** Teste de $t$ Student para variâncias diferentes; IMC=Índice de Massa Corporal.

\section{Tabela 3}

Distribuição de freqüência, prevalência, razão de prevalência bruta para ansiedade das narráveis maternas. Município do Rio de Janeiro, 2005 a 2007.

\begin{tabular}{|c|c|c|c|c|c|c|}
\hline \multirow[t]{3}{*}{ Variáveis } & & \multicolumn{5}{|c|}{ Ansiedade traço* } \\
\hline & \multirow[t]{2}{*}{$\mathrm{N}$} & \multicolumn{2}{|c|}{ Prevalência } & \multirow[t]{2}{*}{$\mathrm{RP} * *$} & \multirow[t]{2}{*}{ IC95\% } & \multirow{2}{*}{ Valor $p^{* * *}$} \\
\hline & & $\mathrm{n}$ & $\%$ & & & \\
\hline \multicolumn{7}{|l|}{ Sócio-demográficas } \\
\hline \multicolumn{7}{|l|}{ Idade (anos) } \\
\hline $18,0+25$ & 83 & 60 & 72,3 & 1,29 & $1,01-1,66$ & 0,036 \\
\hline $25,0+41$ & 68 & 38 & 55,9 & 1,00 & - & \\
\hline \multicolumn{7}{|c|}{ Renda (salário mínimo) $* * * *$} \\
\hline$<1$ & 14 & 8 & 57,1 & 0,91 & $0,76-1,10$ & 0,402 \\
\hline $1-2$ & 84 & 58 & 69,0 & 0,96 & $0,80-1,15$ & \\
\hline $3-5$ & 41 & 23 & 56,1 & 0,74 & $0,49-1,15$ & \\
\hline$\geq 6$ & 12 & 9 & 75,0 & 1,00 & - & \\
\hline \multicolumn{7}{|l|}{ Escolaridade (anos) } \\
\hline $1-8$ & 63 & 49 & 77,8 & 1,35 & $1,08-1,68$ & 0,010 \\
\hline$\geq 9$ & 85 & 49 & 57,6 & 1,00 & - & \\
\hline \multicolumn{7}{|l|}{ Estado marital } \\
\hline Casada & 30 & 17 & 56,7 & 1,08 & $0,70-1,66$ & 0,033 \\
\hline Vive em união & 78 & 58 & 74,4 & 1,19 & $1,02-1,40$ & \\
\hline Não vive em união & 42 & 22 & 52,4 & 1,00 & - & \\
\hline \multicolumn{7}{|l|}{ Cor da pele (observada) } \\
\hline Branca & 42 & 26 & 61,9 & 1,00 & - & 0,687 \\
\hline Preta & 107 & 70 & 65,4 & 1,06 & $0,80-1,39$ & \\
\hline \multicolumn{7}{|c|}{ Trabalho durante a gestação } \\
\hline Sim & 89 & 54 & 60,7 & 1,00 & - & 0,192 \\
\hline Não & 62 & 44 & 71,0 & 1,17 & $0,93-1,47$ & \\
\hline
\end{tabular}


Distribuição de freqüência, prevalência, razão de prevalência bruta para ansiedade das narráveis maternas. Município do Rio de Janeiro, 2005 a 2007

\begin{tabular}{|c|c|c|c|c|c|c|}
\hline \multirow[t]{3}{*}{ Variáveis } & & \multicolumn{5}{|c|}{ Ansiedade traço* } \\
\hline & \multirow[t]{2}{*}{$\mathrm{N}$} & \multicolumn{2}{|c|}{ Prevalência } & \multirow[t]{2}{*}{$R P * *$} & \multirow[t]{2}{*}{ IC95\% } & \multirow[t]{2}{*}{ Valor $p^{* * *}$} \\
\hline & & $\mathrm{n}$ & $\%$ & & & \\
\hline \multicolumn{7}{|l|}{ Obstétricas } \\
\hline \multicolumn{7}{|c|}{ Aborto prévio } \\
\hline Sim & 66 & 45 & 68,2 & 1,09 & $0,87-1,38$ & 0,457 \\
\hline Não & 85 & 53 & 62,4 & 1,00 & - & \\
\hline \multicolumn{7}{|c|}{ Baixo peso ao nascer (prévio) } \\
\hline Sim & 9 & 7 & 77,8 & 1,14 & $0,78-1,68$ & 0,552 \\
\hline Não & 72 & 49 & 68,1 & 1,00 & - & \\
\hline \multicolumn{7}{|c|}{ Prematuridade (prévia) } \\
\hline Sim & 9 & 5 & 55,6 & 1,00 & - & 0,321 \\
\hline Não & 74 & 53 & 71,6 & 1,30 & $0,70-2,36$ & \\
\hline \multicolumn{7}{|l|}{ Álcool } \\
\hline Sim & 22 & 16 & 72,7 & 1,14 & $0,86-1,53$ & 0,405 \\
\hline Não & 129 & 82 & 63,6 & 1,00 & - & \\
\hline \multicolumn{7}{|l|}{ Fumo } \\
\hline Sim & 18 & 14 & 77,8 & 1,23 & $0,93-1,63$ & 0,223 \\
\hline Não & 133 & 84 & 63,2 & 1,00 & - & \\
\hline \multicolumn{7}{|c|}{ Estado nutricional materno pré-gestacional } \\
\hline \multicolumn{7}{|c|}{ IMC pré-gestacional (kg/m²) } \\
\hline$\leq 25,9$ & 108 & 69 & 63,9 & 1,00 & - & 0,680 \\
\hline$\geq 26,0$ & 43 & 29 & 67,4 & 1,06 & $0,82-1,36$ & \\
\hline
\end{tabular}

* Ansiosas - IDATE (Inventário de Ansiedade Traço-Estado) $>40 ; * *$ RP=razão de prevalência bruta; *** Valor de $p$ referente à $\mathrm{RP} ; * * * *$ salário mínimo da época $=\mathrm{R} \$ 350,00$

Tabela 4

Variáveis associadas à ansiedade materna, segundo análise de regressão de Poisson. Município do Rio de Janeiro, 2005 a 2007.

\begin{tabular}{lcc}
\hline Variáveis & \multicolumn{2}{c}{ Ansiedade traço* } \\
\cline { 2 - 3 } & $\mathrm{RP} * *$\begin{tabular}{l} 
IC95\% Valor $p$ \\
\hline Idade (anos)
\end{tabular} & \\
$18,0-24,9$ & 1,35 & $1,06-1,71$ \\
$25,0-41,0$ & 1,00 & - \\
Escolaridade (anos) & & 0,015 \\
$1-8$ & 1,39 & $1,11-1,73$ \\
$\geq 9$ & 1,00 & -
\end{tabular}

*Ansiosas - IDATE (Inventário de Ansiedade Traço-Estado)>40; ** RP=Razão de prevalência ajustada. 


\section{Discussão}

Os resultados do presente estudo revelaram, que a ansiedade traço esteve associada com a idade e a escolaridade. Observou-se risco $35 \%$ maior para gestantes mais jovens e $40 \%$ maior para as de menor escolaridade. Esses resultados estão coerentes com a literatura.6,20 Grimstad et al. ${ }^{20}$ constataram que gestantes mais jovens (idade média de 25 anos) são mais ansiosas do que gestantes mais velhas. Tal associação pode ser parcialmente explicada pela menor maturidade de mães jovens no enfrentamento das dificuldades na gestação. ${ }^{6}$ No que diz respeito à escolaridade, o presente estudo mostrou que o transtorno de ansiedade é mais freqüente em gestantes com menor nível educacional. Segundo Maragno et al.,21 a baixa escolaridade influencia a capacidade de solucionar problemas, afetando os quadros ansiógenos. São raros, contudo, os estudos que enfocam essa relação.

A escala IDATE, aqui empregada, foi validada em estudo nacional que comparou estudantes universitários, pacientes com síndrome do pânico e depressão. A prevalência de ansiedade traço, segundo o estudo de validação, foi de $52,8 \%$ para pacientes ansiosos e $40,7 \%$ para o grupo de estudantes. 12

No presente estudo, observou-se prevalência de ansiedade de $64,9 \%$ valor muito próximo ao encontrado na investigação realizada por Baptista et al.,22 de $65,9 \%$. É importante mencionar, entretanto, que apesar de ambos os estudos terem utilizado o Inventário de Ansiedade traço-estado, os pontos de corte utilizados foram diferentes; 40 neste estudo e 34 a 54 pontos no estudo de Baptista et al.22 Se o presente estudo tivesse utilizado ponto de corte semelhante (49 pontos), 12 a prevalência observada seria mais baixa, de $34,4 \%$. Já o estudo realizado por Freitas e Botega, ${ }^{23}$ ao avaliarem a sintomatologia ansiosa em 120 adolescentes grávidas atendidas em um serviço de pré-natal em São Paulo, utilizando a escala Hospitalar de Ansiedade e Depressão (HAD) e a escala de ideação suicida de Beck, observaram prevalência de ansiedade de 23,3\%. Resultados semelhantes foram observados na investigação conduzida por D'el Rey et al.,24 com 25 gestantes internadas em um hospital de São Paulo, com idades entre 20-35 anos, quando se verificou prevalência de sintomas de ansiedade em $24 \%$ dos casos segundo a HAD.

A alta prevalência de ansiedade traço encontrada no presente estudo $(64,9 \%)$ pode ser explicada parcialmente pela associação com a depressão. A concomitância entre ansiedade e depressão é consi- derada uma das dificuldades na avaliação da ansiedade, já que é impossível caracterizar determinados sintomas que expressem apenas a depressão ou apenas a ansiedade. Sugere-se que os dois construtos possam ser componentes de um processo de estresse psicológico geral.25,26 Por outro lado, considera-se que apesar do risco de sobreposição de sintomas de ansiedade e depressivos com o uso do IDATE, o instrumento fornece adequada medida dos níveis de ansiedade em gestantes. ${ }^{6}$

Algumas limitações do estudo devem ser mencionadas. A primeira refere-se ao ponto de corte empregado para avaliar ansiedade. É possível que tenham sido incluídos falsos positivos e conseqüentemente super estimativa da prevalência de ansiedade. É interessante mencionar que, embora o escore fornecido pelo IDATE seja uma variável contínua, análises de regressão linear não revelaram fatores associados. Esses resultados sugeriram como estratégia alternativa o emprego de um ponto de corte; dessa forma foi selecionado o valor 40, já empregado nos estudos de Rondo et al. 17 e Teixeira et al. $18 \mathrm{O}$ fato de ter sido usada uma escala de auto-avaliação e não critérios psiquiátricos para a classificação dos casos de ansiedade também pode ter contribuído para os elevados valores de prevalência observados. A prevalência de transtornos de ansiedade provavelmente seria menor se fossem empregados critérios clínicos de diagnóstico. Uma outra limitação diz respeito às perdas de seguimento, que foram de $38,8 \%$ para o segundo ponto de observação. Observou-se perda seletiva para a variável estado marital, e a estratégia de controle foi incluir essa variável no modelo final. É importante mencionar que estratégias de controle de perdas foram implementadas, permitindo que ao final do seguimento as perdas diminuíssem para menos de $30 \%$.

$\mathrm{O}$ adequado conhecimento dos motivos que levam a mulher a estar ansiosa, como por exemplo a saúde do bebê, medo do parto, entre outros, pode ser útil para diferenciar os sintomas de ansiedade que seriam considerados esperados para o período, daqueles que necessitariam de um acompanhamento clínico. ${ }^{6}$ Embora os resultados dos estudos sejam, por vezes, contraditórios e ainda insuficientes, há sugestões documentadas de que fatores psicológicos podem acarretar complicações durante a gestação, o parto e o puerpério, bem como para o concepto. Particularmente, um desses fatores pode ser a ansiedade, atuando principalmente durante a gravidez. Dessa forma, o conhecimento dos fatores associados à ansiedade é fundamental para implantação de programas preventivos, em atenção primária, e de suporte, em atenção secundária à saúde. 
Em resumo, observou-se que há uma multiplicidade de instrumentos para o rastreamento da ansiedade em gestantes e que essa diversidade dificulta a comparação das prevalências em diferentes subgrupos. Observou-se ainda alta prevalência de ansiedade, segundo o IDATE, em mulheres atendidas em

\section{Referências}

1. Andersson L, Sundstrom-Poromaa I, Bixo M, Wulff M, Bondestam K, Astrom MM. Point prevalence of psychiatric disorders during the second trimester of pregnancy: a population-based study. Am J Obstet Gynecol. 2003; 189: 14854.

2. Conde F, Figueiredo B. Ansiedade na gravidez: implicações para a saúde e desenvolvimento do bebê e mecanismos neurofisiológicos envolvidos. Acta Pediatr. Port. 2005, 36 41-9.

3. Rini CK, Dunkel-Schetter C, Wadhwa PD, Sandman CA. Psychological adaptation and birth outcomes: the role of personal resources, stress and sociocultural context in pregnancy. Health Psychol. 1999; 18: 333-45.

4. Falcone VM, Mäder CVN, Nascimento CFL, Santos JMM, Nóbrega FJ. Atuação multiprofissional e a saúde mental de gestantes. Rev Saúde Pública. 2005; 39: 171-8.

5. Camacho RS, Cantinelli FS, Ribeiro CS, Cantilino A, Gonsales BK, Braguittoni E, Rennó Jr J. Transtornos psiquiátricos na gestação e no puerpério: classificação, diagnóstico e tratamento. Rev Psiquiatr Clin. 2006; 33: 92 102 .

6. Faisal-Cury A, Menezes PR. Ansiedade no puerpério: prevalência e fatores de risco. Rev Bras Ginecol Obstet. 2006; $28: 171-8$

7. Zimmer-Gembeck MJ, Helfand M. Low birth-weight in a public prenatal care program: behavioral and psychosocial risk factors and psychosocial intervention. Soc Sci Med. 1996; 43: 187-97.

8. Wadhwa PD, Sandman CA, Porto M, Shetter-Dunkel C, Garite TJ. The association between prenatal stress and infant birth weight and gestational age at birth: a prospective investigation. Am J Obstet Gynecol. 1993; 169: 85865.

9. Consonni EB. Repercussões de um programa multidisciplinar de preparo para o parto e maternidade: ansiedade materna e resultados perinatais. Rev Bras Ginecol Obstet. 2002; 24: 205 .

10. Araújo DMR, Pereira NL, Kac G. Ansiedade na gestação, prematuridade e baixo peso ao nascer: uma revisão sistemática da literatura. Cad Saúde Pública. 2007; 23: 74756.

11. Spielberguer CD, Gorsuch RL, Lushene RE. Manual for the state-trait anxiety inventory (self-evaluation questionnaire). Menolo Park, CA: Consulting Psychologists; 1970. uma unidade básica de saúde do município do Rio de Janeiro. Adicionalmente, verificou-se um risco $35 \%$ maior em gestantes mais jovens de apresentarem ansiedade e $40 \%$ para as de menor escolaridade.

12. Biaggio AMB, Natalício L, Spielberguer CD Desenvolvimento da forma experimental em português do Inventário de ansiedade traço-estado (IDATE) de Spielberguer. Arq Bras Psicol Apl. 1977; 29: 31-44.

13. Cattell RB, Scheier IH. The meaning and measurement of neuroticism and anxiety. New York, Ronald Press; 1961.

14. Biaggio AMB, Natalício L. Manual do IDATE. Rio de Janeiro: CEPA; 1979.

15. La Rosa, J. Inventário de ansiedade traço-estado: características psicométricas. Est Psicol. 10: 81-92, 1993.

16. Newton RW, Hunt LP. Psychosocial stress in pregnancy and its relation to low birth weight. BMJ. 1984; 288: 1191-4.

17. Rondo PHC, Ferreira RF, Nogueira F, Ribeiro MCN, Lobert $\mathrm{H}$, Artes R. Maternal psychological stress and distress as predictors of low birth weight, prematurity and intrauterine growth retardation. Eur J Clin Nutr. 2003; 57: 266-72.

18. Teixeira J, Fisk N, Glover V. Association between maternal anxiety in pregnancy and increased uterine artery resistance index: cohort based study. BMJ. 1999; 16: 153-7.

19. Barros AJD, Hirakata VN. Alternatives for logistic regression in cross-sectional studies: an empirical comparison of models that directly estimate the prevalence ratio. BMC Med Res Methodol. 2003; 3: 21.

20. Grimstad H, Schei B, Backe B, Jacobsen G. Anxiety, physical abuse, and low birth weight. Scand J Public Health. 1999; 27: 296-300.

21. Maragno L, Goldbaum M, Gianini RJ, Novaes HMD, César CLC. Prevalência de transtornos mentais comuns em populações atendidas pelo Programa Saúde da Família (QUALIS) no Município de São Paulo. Cad Saúde Pública. 2006; 22: 1639-48.

22. Baptista MN, Baptista ASD, Torres ECR. Associação entre suporte social, depressão e ansiedade em gestantes. Rev Psicol. 2006; 7: 39-48.

23. Freitas GVS, Botega NJ. Gravidez na adolescência: prevalência de depressão, ansiedade e ideação suicida. Rev Assoc Med Bras. 2002; 48: 1-10.

24. D'el Rey GJF, Quayle J, Lúcia MCS, Zugaib M. Aspectos cognitivos relacionados ao aparecimento de transtornos de humor em gestantes internadas. Rev Ginecol Obstet. 2000; 11: 181-5.

25. Andrade LHSG, Gorestein C. Aspectos gerais das escalas de avaliação de ansiedade. Rev Psiquiatr Clin. 1998; 25: $285-90$

26. Nardi AE. Comentários do debatedor: escalas de avaliação de ansiedade. Rev Psiq Clin. 1998; 25: 331-3.

Recebido em 19 de setembro 2006

Versão final apresentada em 9 de outubro de 2007

Aprovado em 12 de dezembro de 2007 